\title{
咔唑基高价碘试剂参与的活化芳烃直接咔唑化反应研究
}

\author{
兰天䂞 $a, b$ 张 越 ${ }^{a, b}$ 刘伟 $c$ 席婵娟 $a$ 陈 超*, $a$ \\ ( ${ }^{a}$ 清华大学化学系 生命有机磷化学及化学生物学教育部重点实验室 北京 100084) \\ ( ${ }^{b}$ 天津科技大学化工与材料学院 天津 300457) \\ ( ${ }^{c}$ 天津科技大学理学院 天津 300457 )
}

\begin{abstract}
摘要 咔唑及其衍生物在医药和光电材料领域有着广泛的应用. 合成了一种稳定的含有咔唑基团的环状高价碘试剂, 该试剂属于苯并碘氧杂环类化合物，在 $\mathrm{Cu}(\mathrm{I})$ 催化条件下可以与芳烃底物反应，得到 $N$-芳基咔唑衍生物，反应条件温和， 适用于多种富电子芳烃，并提出了一个可能的自由基反应机理.
\end{abstract}

关键词 咔唑; 高价碘试剂; $N$-芳基化; 铜催化

\section{Carbazolation Study of Active Arenes with Carbazole-Containing Hypervalent lodine(III) Reagents}

\author{
Lan, Tianlei ${ }^{a, b} \quad$ Zhang, Yue ${ }^{a, b} \quad{\text { Liu, } \text { Wei }^{c} \quad \mathrm{Xi}, \text { Chanjuan }}^{a} \quad$ Chen, Chao ${ }^{*, a}$ \\ $\left({ }^{a}\right.$ Key Laboratory of Bioorganic Phosphorus Chemistry \& Chemical Biology, Ministry of Education, \\ Department of Chemistry, Tsinghua University, Beijing 100084) \\ ( ${ }^{b}$ College of Chemical Engineering and Materials Science, Tianjin University of Science \& Technology, Tianjin 300457) \\ ( ${ }^{c}$ College of Sciences, Tianjin University of Science \& Technology, Tianjin 300457)
}

\begin{abstract}
Carbazole and its derivatives are widely used in the field of medicine and photoelectric materials. A kind of stable cyclic hypervalent iodine reagents containing carbazole group was developed, which belong to benziodoxole compounds. In the presence of $\mathrm{Cu}(\mathrm{I})$ catalyst, these reagents reacted with aromatic substrates to give $N$-aryl carbazole derivatives. The reaction conditions are mild and suitable for a variety of electron-rich arenes. And a radical mechanism was proposed.

Keywords carbazole; hypervalent iodine reagent; $N$-arylation; copper-catalysis
\end{abstract}

咔唑分子是许多天然产物分子中非常重要的有机 砌块之一, 咔唑类生物碱具有良好的生物活性和药理学 特性, 例如抗炎、抗癌、抗病毒和抗氧化等 ${ }^{[1]}$. 此外, 咔唑类化合物具有独特的光电性能, 是现代材料科学的 一个重要的研究方向, 咔唑及其衍生物目前被广泛应用 于小分子和聚合物有机发光二极管(OLEDs) 以及太阳能 电池等领域(图 1) ${ }^{[2]}$.

$N$-芳基咔唑衍生物是一类在光电材料领域有着重 要应用的有机分子, 传统的合成方法是通过 Ullmann 或 Buchwald-Hartwig 反应构筑 $\mathrm{C}-\mathrm{N}$ 键得到 ${ }^{[3]}$, 但是这类 反应需要苛刻的反应条件, 例如高温、使用强碱和昂贵
的催化剂，并且需要使用芳基(拟)卤化物作为底物，而 利用廉价的反应原料和温和的反应条件合成 $N$-芳基咔 坐衍生物仍然是一项具有挑战性的任务.

高价碘化合物由于其非经典的化学键性质和特殊 的反应活性, 一直吸引着合成化学家的关注 ${ }^{[4]}$. 在近年 来, 苯并碘氧杂环类环状高价碘试剂, 因其在官能团转 移反应中的优异性能, 已经得到了广泛的研究 ${ }^{[5]}$. 其中, 三氟甲基(Togni reagents) ${ }^{[6]}$ 、炔基(EBXs) ${ }^{[7]}$ 和叠氮基

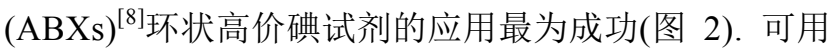
于氮原子转移的高价碘试剂除了 ABXs 试剂外, 最近, Minakata 课题组 ${ }^{[9]}$ 和 Bolm 课题组 ${ }^{[10]}$ 分别报道了含有邻

\footnotetext{
* Corresponding author. E-mail: chenchao01@mails.tsinghua.edu.cn

Received May 28, 2019; revised July 12, 2019; published online July 17, 2019.

Dedicated to the 100th anniversary of the birth of Professor Ruyu Chen.

Project supported by the National Key Research and Development Program of China (No. 2016YFB0401400), the National Natural Science Foundation of China (Nos. 21302139, 21672120, 21871158) and the Fok Ying Tong Education Foundation of China (No. 151014).

国家重点研发计划(No. 2016YFB0401400)、国家自然科学基金(Nos. 21302139, 21672120, 21871158)和霍英东青年教师基金(No. 151014)资助项目.
} 
<smiles>c1ccc2c(c1)c1ccccc1n2-c1ccc(-c2ccc(-n3c4ccccc4c4ccccc43)cc2)cc1</smiles>

CBP<smiles></smiles>

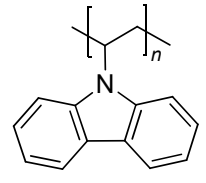

PVK
图 1 咔唑衍生物

Figure 1 Carbazole derivatives

苯二甲酰亚胺和磺酰胺等含氮基团的环状高价碘试剂.<smiles>O=C1OI(C(F)(F)F)c2ccccc21</smiles>

Togni reagent<smiles>Nc1ccccc1C(=O)O</smiles>

$\mathrm{ABX}$<smiles>[R]C#CI1COC(=O)c2ccccc21</smiles>

EBX<smiles>O=C1OI([N+]=Nc2ccccc2)c2ccccc21</smiles>

Minakata's work<smiles>[R]S([R])(=O)=NI1COC(=O)c2ccccc21</smiles>

Bolm's work
图 2 苯并碘氧杂环类化合物

Figure 2 Benziodoxol(on)e compounds

在此研究背景下, 结合我们课题组前期的研究工 作 ${ }^{[11]}$, 开发了一类基于苯并碘氧杂环结构的含有咔唑基 团的高价碘试剂, 进一步利用这种试剂实现了在温和条 件下的 $N$-芳基咔唑衍生物的合成. 在本工作中, 我们用 乙酰氧基环状高价碘化合物和 $N$-TMS-咔唑为原料, 能 够以良好的收率得到一系列稳定的咔唑基环状高价碘 试剂(Scheme 1). 并且, 在一价铜的催化作用下, 这种 新型高价碘试剂可以与活化的芳香底物反应, 以中等至 良好的收率得到相应的咔唑衍生物。

\section{1 结果与讨论}

\section{1 咔唑基环状高价碘试剂}

首先, 对含咔唑基的环状高价碘试剂的合成方法进 行了探究. 我们在前期的研究工作中已经发现, 当选用 高价碘化合物 $\mathbf{1}$ 与 $N$-TMS-咔唑衍生物 $\mathbf{2}$ 作为原料, 干 燥的乙腈作溶剂, 催化量的氟化钾作添加剂, 在室温下 反应，能够成功得到含有咔唑基团的目标高价碘化合物 3(表 1). 当使用咔唑的芳环没有被其它原子修饰的底物
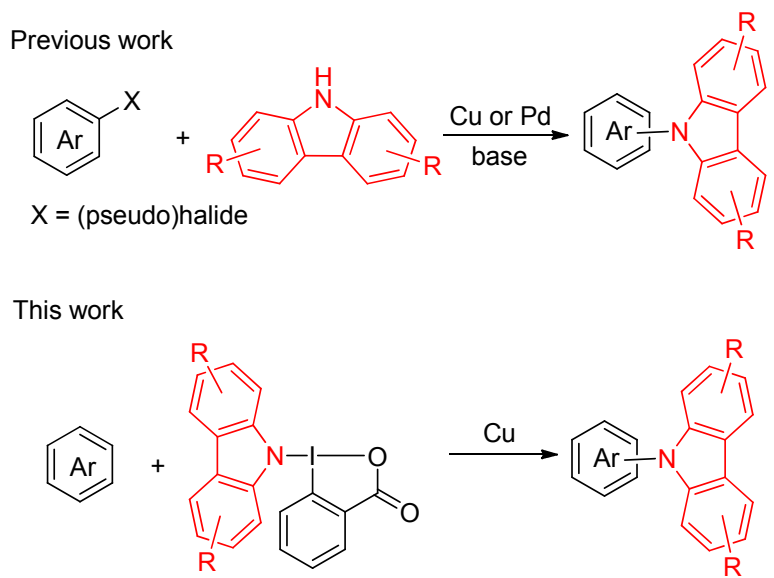

图式 $1 \mathrm{~N}$-芳基咔唑衍生物的合成

Scheme 1 Synthesis of $N$-arylated carbazoles

进行反应时，相应的目标产物 3a 的收率最高，为 $91 \%$; 当咔唑底物的 3 位和 6 位的氢原子被卤素原子取代时, 能以 $55 \% \sim 68 \%$ 的收率获得相应的目标产物. 此外，当 咔唑的 2 位和 7 位的氢原子被澳原子取代时，目标产物 3f 也能够被顺利得到. 但是，当咔唑带有甲氧基或硝基 时, 不能得到相应的高价碘产物. 此类咔唑基环状高价 碘化合物在空气中稳定，常温下几乎不溶于乙腈，但可 以溶于二氯甲烷、氯仿和二甲基亚砜等有机溶剂.

表 1 高价碘试剂的拓展 ${ }^{a}$

Table 1 Scope of hypervalent iodine reagents

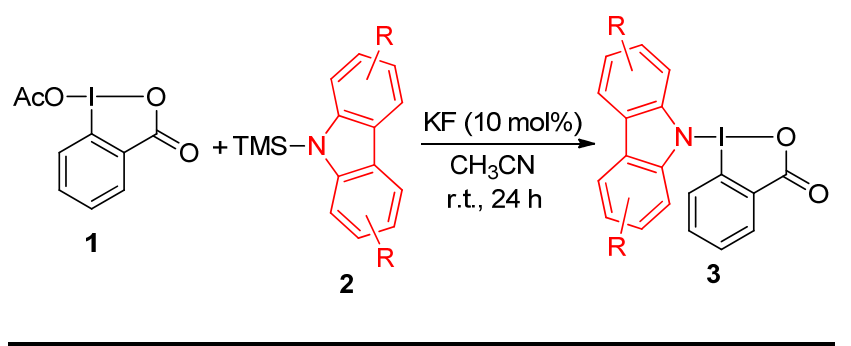

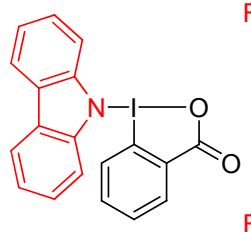

3a: $91 \%$

3d: $65 \%$

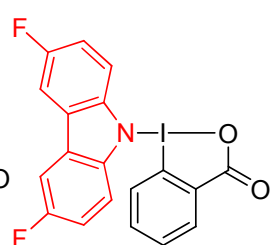

3b: $68 \%$

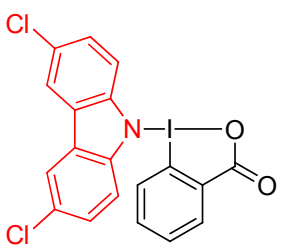

3c: $55 \%$<smiles>O=C1O[IH]2(OC(=O)N2c2ccc(I)cc2-n2c3ccc(Br)cc3c3cc(I)ccc32)c2ccccc21</smiles>
${ }^{a}$ Reaction conditions: $1(4.0 \mathrm{mmol}), 2(4.0 \mathrm{mmol}), \mathrm{KF}(10 \mathrm{~mol} \%)$, in $\mathrm{CH}_{3} \mathrm{CN}$ $(10 \mathrm{~mL})$ at room temperature. Isolated yields. 


\section{2 高价碘试剂参与的 $N$-芳基咔唑衍生物的合成}

根据前期的研究结果, 我们发现咔唑基环状高价碘 试剂在以 $(\mathrm{CuOTf})_{2} \mathrm{C}_{6} \mathrm{H}_{6}$ 作催化剂, 乙腈为溶剂, 反应温 度为 $80{ }^{\circ} \mathrm{C}$ 的标准条件下具有良好的反应性，可以与各 类富电子的芳烃底物发生自由基取代反应, 得到相应的 $N$-芳基化咔唑衍生物(表 2).

首先使用稠环芳烃萠 $\mathbf{4 a}$ 分别与咔唑基环状高价碘 试剂 3a 3f 在标准条件下反应, 可以分离得到萠的 1 位 氢原子被咔唑基团取代的目标产物 $\mathbf{5 a a} \sim 5 \mathbf{a f}$, 收率为 $43 \% \sim 65 \%$. 随后，我们使用高价碘试剂 3d 为模板，考 察其它芳烃底物在该反应体系中的适用性. 使用菜作为 底物时, 目标产物 $5 \mathbf{b}$ 收率为 $40 \%$; 当在萗环的 2 位上带 有给电子基团时, 能够以中等的收率选择性地得到 1 位 被咔唑基团取代的产物 $\mathbf{5 c} \sim \mathbf{5 f}$ ，此外，使用苊作底物时，
可以得到 5 位被咔唑基团取代的目标产物 $5 \mathrm{~g}$, 收率为 $61 \%$. 菲或蒽类底物的反应性与菜相似, 在标准条件下 可以顺利得到在菲或蒽的 9 位被咔唑基团取代的产物; 特别地，使用带有吸电子基团的 9-溴菲作为底物时，咔 唑基团可以顺利地被引入溴原子的邻位，得到目标产物 5i. 进一步地，尝试使用非稠环芳香底物在该体系下进 行反应，当选用富电子芳烃底物 $4 \mathrm{n}$ 和 40 与咔唑高价碘 试剂反应时，可以得到相应的目标产物 $5 n$ 和 5o. 参考 我们课题组前期的工作，由于该反应经历自由基过程， 可以产生氮中心的咔唑自由基，进而生成咔唑分子经 $\mathrm{N}$ - $\mathrm{N}$ 偶联的副产物，降低了产物的收率. 此外，当芳烃 底物带有强吸电子基团，例如硝基、氰基、三氟甲基或 酯基时，反应不能够顺利进行.

表 2 芳烃底物的拓展 ${ }^{a}$

Table 2 Scope of arenes

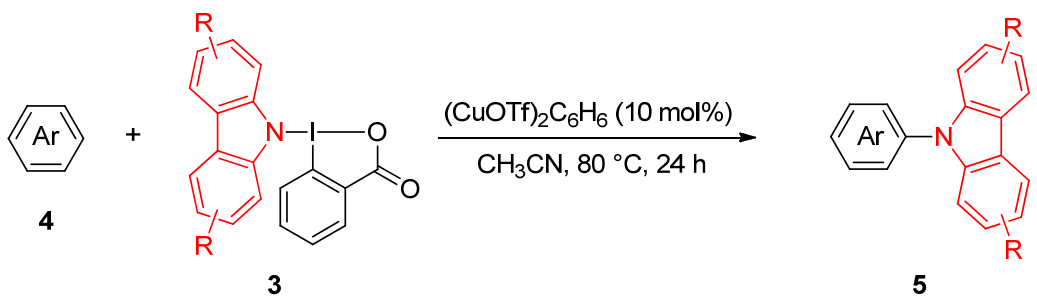

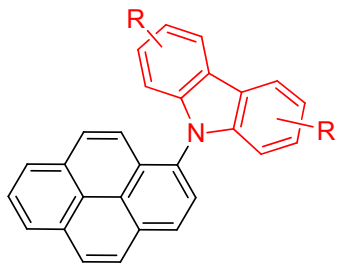

5aa, $\mathrm{R}=\mathrm{H}, 59 \%$; $5 \mathrm{ab}, \mathrm{R}=3,6-\mathrm{F}_{2}, 62 \%$ 5ac, $\mathrm{R}=3,6-\mathrm{Cl}_{2}, 65 \%$; 5ad, $\mathrm{R}=3,6-\mathrm{Br}_{2}, 65 \%$ 5ae, $R=3,6-I_{2}, 43 \% ;$ aaf, $R=2,7-\mathrm{Br}_{2}, 55 \%$

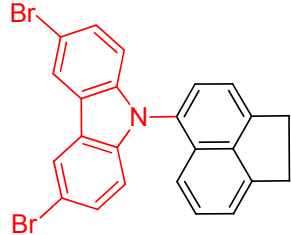

$5 g, 61 \%$

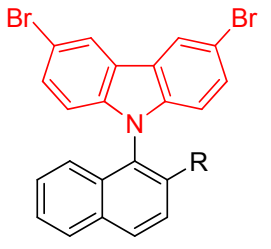

$5 b, \mathrm{R}=\mathrm{H}, 40 \%$ 5c, $\mathrm{R}=\mathrm{MeO}, 48 \%$

5d, $R=$ MeS, $34 \%$

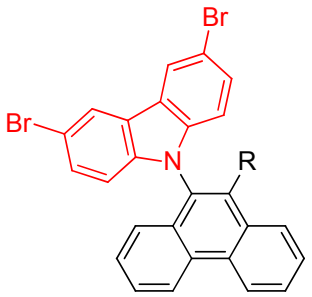

5h, $\mathrm{R}=\mathrm{H}, 69 \%$ 5i, $\mathrm{R}=\mathrm{Br}, 37 \%$

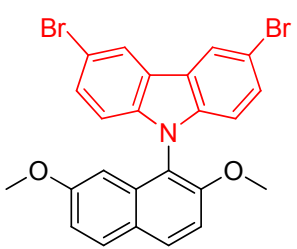

5 e $50 \%$<smiles>COc1ccc2cc(Br)ccc2c1-n1c2ccc(Br)cc2c2cc(Br)ccc21</smiles>

5f, $69 \%$

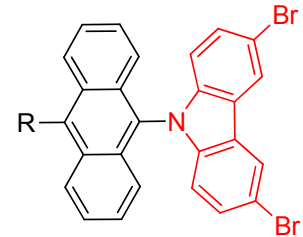

5j, $R=\mathrm{H}, 38 \%$ 5k, R $=\mathrm{Ph}, 62 \%$

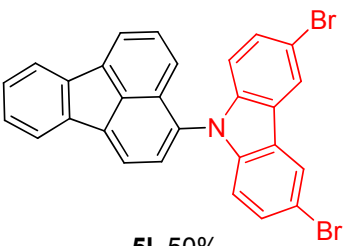

$5 \mathbf{I}, 50 \%$

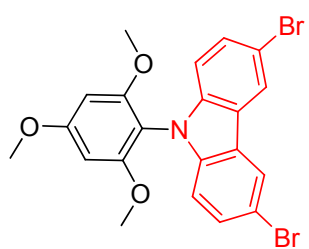

5o, $19 \%$

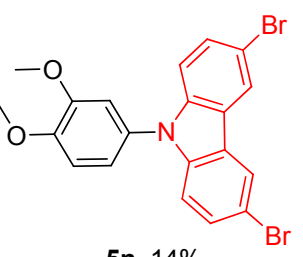

5n, $14 \%$

$5 \mathrm{~m}, 22 \%$

${ }^{a}$ Reaction conditions: $3(0.3 \mathrm{mmol}), 4(0.2 \mathrm{mmol}),(\mathrm{CuOTf}){ }_{2} \mathrm{C}_{6} \mathrm{H}_{6}(10 \mathrm{~mol} \%)$, in $\mathrm{CH}_{3} \mathrm{CN}(4 \mathrm{~mL})$ at $80{ }^{\circ} \mathrm{C}$ for $24 \mathrm{~h}$. Isolated yield. 
咔唑基环状高价碘试剂不仅可以与富电子的芳烃 底物进行反应，还可以与三苯胺及咔唑衍生物等含氮底 物进行反应，顺利地将咔唑基团引入至底物分子中(表 3). 三苯胺与高价碘试剂 3a、3c 和 3d 反应, 以良好的 收率得到产物 5pa 5pc; 但含有 2,7-二溴咔唑基团的产 物收率偏低, 只有 $11 \%$. 4-溴三苯胺 $\mathbf{4 q}$ 与高价碘试剂 $\mathbf{3 d}$ 反应时, 以 $48 \%$ 的收率得到目标产物 5q. 特别地, 当使 用咔唑衍生物 $4 r \sim 4 t$ 作为底物时, 以中等的分离收率得 到二咔唑产物 $5 r \sim 5 t$; 如果咔唑底物含有 $\mathrm{N}-\mathrm{H}$ 键则不 能发生该反应. 上述几个例子表明, 咔唑基环状高价碘 试剂可以与咔唑类底物发生反应, 在咔唑的 3 或 6 位再 引入一个咔唑分子. 因此, 通过该方法得到的 $N$-芳基咔

表 3 含氮底物的拓展 ${ }^{a}$

Table 3 Scope of nitrogenous substrates
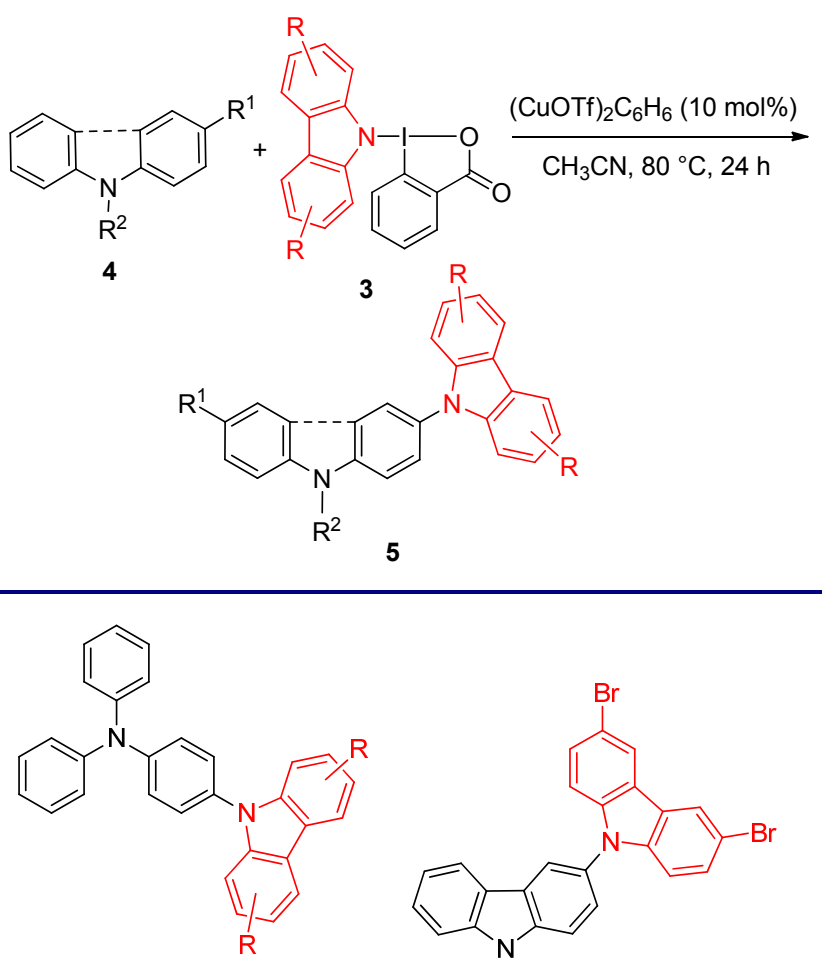

5pa, $\mathrm{R}=\mathrm{H}, 61 \%$

$5 \mathrm{pb}, \mathrm{R}=3,6-\mathrm{Cl}_{2}, 44 \%$

5pc, $\mathrm{R}=3,6-\mathrm{Br}_{2}, 48 \%$

5pd, $\mathrm{R}=2,7-\mathrm{Br}_{2}, 11 \%$
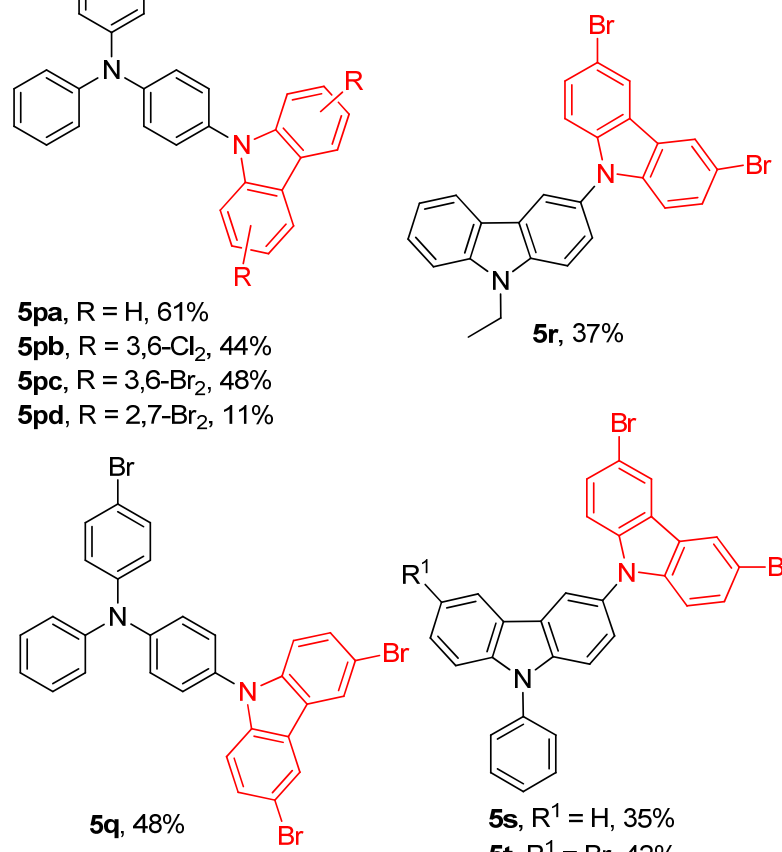

$5 q, 48 \%$

$\mathrm{Br}$

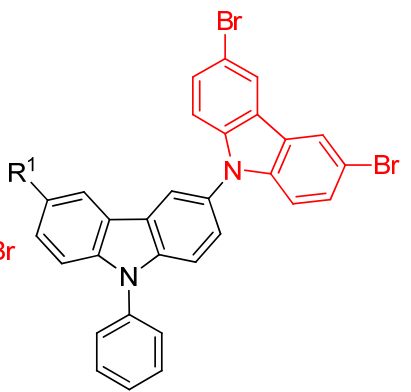

5s, $\mathrm{R}^{1}=\mathrm{H}, 35 \%$

5t, $\mathrm{R}^{1}=\mathrm{Br}, 42 \%$

\footnotetext{
${ }^{a}$ Reaction conditions: $3(0.3 \mathrm{mmol}), \mathbf{4}(0.2 \mathrm{mmol}),(\mathrm{CuOTf})_{2} \mathrm{C}_{6} \mathrm{H}_{6}(10 \mathrm{~mol} \%)$, in $\mathrm{CH}_{3} \mathrm{CN}(4 \mathrm{~mL})$ at $80{ }^{\circ} \mathrm{C}$ for $24 \mathrm{~h}$. Isolated yield.
}

唑衍生物分子可以继续在反应体系中作为底物参与反 应，这可能导致单咔唑取代的目标产物收率偏低.

基于我们课题组 ${ }^{[12]}$ 前期研究工作的结果并参考已 有的文献报道，我们提出了一个可能的反应机理 (Scheme 2). 首先, 咔唑基环状高价碘试剂在一价铜的 作用下发生一个单电子转移反应，产生一个氮中心的咔 唑自由基 $\mathbf{A}$, 并且同时释放出一个二价铜中间体 $\mathbf{B}$. 接 下来，咔唑自由基 $\mathbf{A}$ 与芳烃或芳香杂环类底物发生自由 基加成反应，产生自由基中间体 C. 最后，自由基中间 体 $\mathbf{C}$ 在二价铜中间体 B 的作用下, 发生氧化反应和去质 子化反应, 最终得到相应的目标产物, 并且再生出一个 一价铜物种进入催化循环.

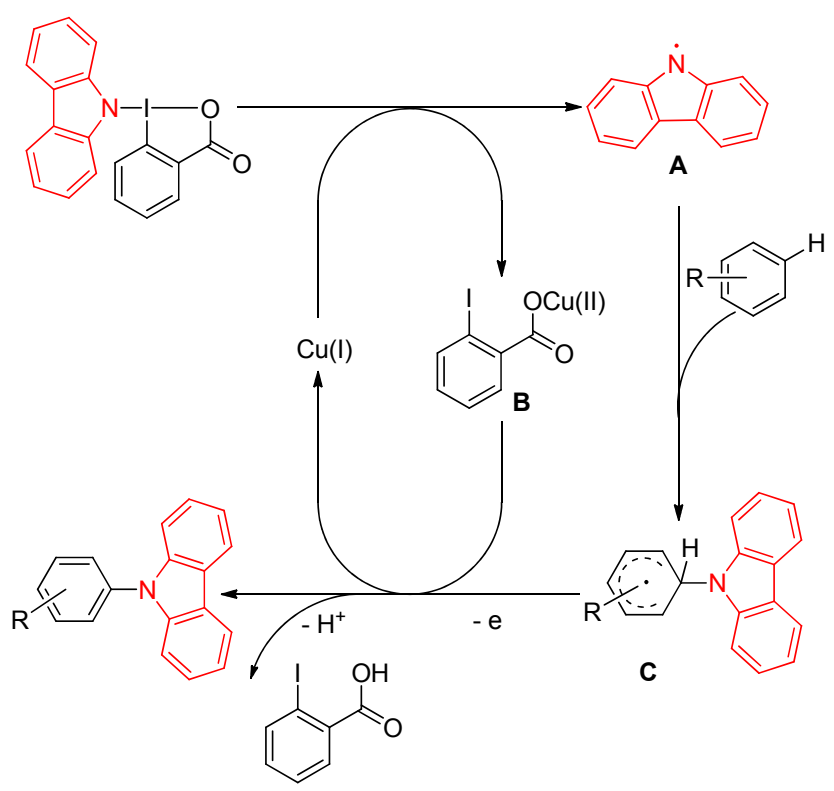

图式 2 可能的反应机理

Scheme 2 Plausible reaction mechanism

\section{2 结论}

利用新型的咔唑基环状高价碘试剂，实现了一系列 $N$-芳基化咔唑衍生物的合成. 与传统的 $\mathrm{C}-\mathrm{N}$ 偶联反应 相比，该反应无需使用预活化的底物以及苛刻的反应条 件，可以在温和的条件下得到含有多个卤素原子的产物 分子, 这对于产物衍生化及应用是十分有利的. 我们相 信这种新型高价碘试剂在有机合成和材料科学领域有 着广阔的应用前景.

\section{3 实验部分}

\section{1 仪器与试剂}

所有反应均使用预干燥的 Schlenk 管在氮气氛围下 进行. 柱层析使用 $200 \sim 300$ 目硅胶(青岛海洋化工工 厂). ${ }^{1} \mathrm{H}$ NMR 和 ${ }^{13} \mathrm{C}$ NMR 使用 JEOL AL-400 MHz 核磁 
共振仪在常温下测定, 以 $\mathrm{CDCl}_{3}$ 或 $\mathrm{DMSO}-d_{6}$ 为溶剂, TMS 为内标. 高分辨质谱使用 Shimadzu LCMS-IT/TOF 质谱仪在正离子模式下测定.

\section{2 实验方法}

\section{2 .1 咔唑基环状高价碘试剂的合成}

称取高价碘化合物 1 (4.0 mmol, 1.0 equiv.), $N$-TMS 咔唑衍生物 2 (4.0 mmol, 1.0 equiv.) 和氟化钾 $(0.4 \mathrm{mmol}$, 0.1 equiv.)放入 Schlenk 管中, 抽换氮气 3 次, 再加入干 燥乙腈 $(10 \mathrm{~mL})$, 在室温下搅拌 $24 \mathrm{~h}$. 反应结束后, 过滤, 收集固体产物并用乙腈洗涤 $(5 \mathrm{~mL} \times 2)$. 再将所得固体 溶于二氯甲烷, 搅拌 $30 \mathrm{~min}$, 过滤, 收集滤液, 旋干溶 剂, 最后再用少量乙醚洗涤, 干燥, 可以得到相应的目 标产物 3.

1-(9-咔唑基)-1,2-苯并碘氧杂环-3-酮(3a) ${ }^{[11]}$ : 黄色 固体, 产率 91\%. m.p. $141 \sim 142{ }^{\circ} \mathrm{C} ;{ }^{1} \mathrm{H}$ NMR $(400 \mathrm{MHz}$, DMSO-d $\left.d_{6}\right) \delta: 8.25(\mathrm{~d}, J=7.8 \mathrm{~Hz}, 2 \mathrm{H}), 8.17(\mathrm{dd}, J=7.4$, $1.6 \mathrm{~Hz}, 1 \mathrm{H}), 7.77 \sim 7.61(\mathrm{~m}, 4 \mathrm{H}), 7.48(\mathrm{t}, J=7.8 \mathrm{~Hz}, 2 \mathrm{H})$, $7.34(\mathrm{t}, J=7.6 \mathrm{~Hz}, 2 \mathrm{H}), 6.67(\mathrm{~d}, J=8.2 \mathrm{~Hz}, 1 \mathrm{H}) ;{ }^{13} \mathrm{C} \mathrm{NMR}$ $\left(101 \mathrm{MHz}, \mathrm{DMSO}-d_{6}\right) \delta: 168.8,145.0,136.2,133.0,132.7$, $131.9,127.5,126.5,125.4,121.9,121.6,121.2,113.9$; ESI-HRMS calcd for $\mathrm{C}_{19} \mathrm{H}_{12} \mathrm{INO}_{2} \mathrm{Na}[\mathrm{M}+\mathrm{Na}]^{+} 435.9805$, found 435.9811 .

1-(3,6-二氟-9-咔唑基)-1,2-苯并碘氧杂环-3-酮(3b): 黄色固体, 产率 $68 \%$. m.p. $129 \sim 130{ }^{\circ} \mathrm{C} ;{ }^{1} \mathrm{H}$ NMR (400 $\left.\mathrm{MHz}, \mathrm{DMSO}-d_{6}\right) \delta: 8.20 \sim 8.11(\mathrm{~m}, 3 \mathrm{H}), 7.77 \sim 7.65(\mathrm{~m}$, 4H), $7.36(\mathrm{td}, J=9.1,2.7 \mathrm{~Hz}, 2 \mathrm{H}), 6.72(\mathrm{~d}, J=8.1 \mathrm{~Hz}$, $1 \mathrm{H}) ;{ }^{13} \mathrm{C}$ NMR (101 MHz, DMSO- $\left.d_{6}\right) \delta: 168.8,158.6(\mathrm{~d}$, $J=235.4 \mathrm{~Hz}$ ), 142.7, 134.7 (d, $J=337.7 \mathrm{~Hz}), 132.5,131.9$, 126.5, 125.8 (d, $J=4.0 \mathrm{~Hz}), 125.7$ (d, $J=4.1 \mathrm{~Hz}), 121.5$, 115.5, 115.5 (d, $J=9.4 \mathrm{~Hz}), 107.7$ (d, $J=24.2 \mathrm{~Hz}$ ); ESI-HRMS calcd for $\mathrm{C}_{19} \mathrm{H}_{10} \mathrm{~F}_{2} \mathrm{INO}_{2}$ 448.9724, found 448.9726.

1-(3,6-二氯-9-咔唑基)-1,2-苯并碘氧杂环-3-酮 $(3 \mathrm{c})^{[11]}$ : 黄色固体, 产率 $55 \%$. m.p. $149 \sim 150{ }^{\circ} \mathrm{C} ;{ }^{1} \mathrm{H}$ NMR (400 MHz, DMSO- $\left.d_{6}\right) \delta: 8.46(\mathrm{~d}, J=2.1 \mathrm{~Hz}, 2 \mathrm{H})$, $8.17(\mathrm{dd}, J=7.7,1.6 \mathrm{~Hz}, 1 \mathrm{H}), 7.76(\mathrm{~d}, J=8.7 \mathrm{~Hz}, 2 \mathrm{H})$, $7.73(\mathrm{~d}, J=7.3 \mathrm{~Hz}, 1 \mathrm{H}), 7.67$ (td, $J=7.7,1.7 \mathrm{~Hz}, 1 \mathrm{H}), 7.53$ $(\mathrm{dd}, J=8.7,2.2 \mathrm{~Hz}, 2 \mathrm{H}), 6.68(\mathrm{~d}, J=8.2 \mathrm{~Hz}, 1 \mathrm{H}) ;{ }^{13} \mathrm{C}$ NMR (101 MHz, DMSO- $\left.d_{6}\right) \delta: 168.8,144.3,136.5,133.0$, 132.3, 132.0, 127.9, 126.5, 126.4, 125.8, 121.7, 121.4, 115.8; ESI-HRMS calcd for $\mathrm{C}_{19} \mathrm{H}_{10} \mathrm{C}_{12} \mathrm{INO}_{2} \quad 480.9128$, found 480.9118 .

1-(3,6-二溴-9-咔唑基)-1,2-苯并碘氧杂环-3-酮 $(3 d)^{[11]}$ : 黄色固体, 产率 65\%. m.p. $198 \sim 199{ }^{\circ} \mathrm{C} ;{ }^{1} \mathrm{H}$ NMR (400 MHz, DMSO- $\left.d_{6}\right) \delta: 8.60(\mathrm{~d}, J=2.0 \mathrm{~Hz}, 2 \mathrm{H})$, $8.17(\mathrm{dd}, J=7.4,1.7 \mathrm{~Hz}, 1 \mathrm{H}), 7.77 \sim 7.61(\mathrm{~m}, 6 \mathrm{H}), 6.65(\mathrm{~d}$, $J=7.9 \mathrm{~Hz}, 1 \mathrm{H}) ;{ }^{13} \mathrm{C}$ NMR (101 MHz, DMSO- $\left.d_{6}\right) \delta: 168.8$, $144.4,136.5,133.0,132.3,132.0,130.5,126.5,126.3$, $124.7,121.3,116.2,114.3$; ESI-HRMS calcd for $\mathrm{C}_{19} \mathrm{H}_{10} \mathrm{Br}_{2} \mathrm{INO}_{2} 570.8098$, found 570.8096 .

1-(3,6-二碘-9-咔唑基)-1,2-苯并碘氧杂环-3-酮 $(3 \mathrm{e})^{[11]}$ : 黄色固体, 产率 $59 \%$. m.p. $151 \sim 152{ }^{\circ} \mathrm{C} ;{ }^{1} \mathrm{H}$ NMR (400 MHz, DMSO- $\left.d_{6}\right) \delta: 8.72(\mathrm{~d}, J=1.7 \mathrm{~Hz}, 2 \mathrm{H})$, $8.16(\mathrm{dd}, J=7.4,1.7 \mathrm{~Hz}, 1 \mathrm{H}), 7.76(\mathrm{dd}, J=8.7,1.9 \mathrm{~Hz}$, 2H), 7.73 (d, $J=7.2 \mathrm{~Hz}, 1 \mathrm{H}), 7.66(\mathrm{td}, J=7.8,1.7 \mathrm{~Hz}$, $1 \mathrm{H}), 7.57$ (d, $J=8.6 \mathrm{~Hz}, 2 \mathrm{H}), 6.62(\mathrm{~d}, J=8.0 \mathrm{~Hz}, 1 \mathrm{H}) ;{ }^{13} \mathrm{C}$ NMR (101 MHz, DMSO- $\left.d_{6}\right) \delta: 168.8,144.4,136.5,135.9$, $133.0,132.3,132.0,130.6,126.7,126.5,121.3,116.5$, 85.6; ESI-HRMS calcd for $\mathrm{C}_{19} \mathrm{H}_{10} \mathrm{I}_{3} \mathrm{NO}_{2} 664.7840$, found 664.7835 .

1-(2,7-二溴-9-咔唑基)-1,2-苯并碘氧杂环-3-酮(3f): 白色固体，产率 56\%. m.p. 183 $184{ }^{\circ} \mathrm{C} ;{ }^{1} \mathrm{H}$ NMR (400 MHz, DMSO- $\left.d_{6}\right) \delta: 8.22(\mathrm{~d}, J=8.2 \mathrm{~Hz}, 2 \mathrm{H}), 8.18(\mathrm{dd}, J=$ 7.3, $1.7 \mathrm{~Hz}, 1 \mathrm{H}), 8.06(\mathrm{~d}, J=1.7 \mathrm{~Hz}, 2 \mathrm{H}), 7.79 \sim 7.64(\mathrm{~m}$, 2H), 7.49 (dd, $J=8.2,1.8 \mathrm{~Hz}, 2 \mathrm{H}), 6.78(\mathrm{~d}, J=8.0 \mathrm{~Hz}$, $1 \mathrm{H}) ;{ }^{13} \mathrm{C}$ NMR (101 MHz, DMSO- $\left.d_{6}\right) \delta: 169.0,146.5$, 136.4 , 133.0, 132.3, 131.9, 126.4, 125.0, 124.0, 123.4, 122.1, 120.8, 117.1; ESI-HRMS calcd for $\mathrm{C}_{19} \mathrm{H}_{10} \mathrm{Br}_{2} \mathrm{INO}_{2}$ 570.8098 , found 570.8098 .

\subsubsection{N-芳基咔唑衍生物的合成}

称取咔唑基环状高价碘试剂 3 (0.2 mmol, 1.0 equiv.)、底物 4 (0.3 mmol, 1.5 equiv.)和 $(\mathrm{CuOTf})_{2} \mathrm{C}_{6} \mathrm{H}_{6}$ (0.02 mmol, 0.1 equiv.) 放入 Schlenk 管中, 抽换氮气 3 次, 再加入乙腈 $(4 \mathrm{~mL})$, 将反应体系升温至 $80{ }^{\circ} \mathrm{C}$, 搅拌反 应 $24 \mathrm{~h}$. 反应结束后, 将反应体系冷却至室温, 向反应 体系加入饱和氯化钠水溶液 $(5 \mathrm{~mL})$ 淬灭反应, 用二氯甲 烷萃取 $(5 \mathrm{~mL} \times 3)$, 然后用无水硫酸钠干燥有机相, 过 滤, 浓缩, 经硅胶色谱柱纯化, 洗脱剂为石油醚/二氯甲 烷 $(V: V=50: 1 \sim 10: 1)$, 分离得到目标产物 5 .

9-(1-萠基)-咔唑(5aa): 白色固体，产率 59\%. m.p. 246 $247{ }^{\circ} \mathrm{C} ;{ }^{1} \mathrm{H}$ NMR (400 MHz, Chloroform-d) $\delta: 8.37$ $(\mathrm{d}, J=7.9 \mathrm{~Hz}, 1 \mathrm{H}), 8.33 \sim 8.25(\mathrm{~m}, 3 \mathrm{H}), 8.23 \sim 8.17(\mathrm{~m}$, $3 \mathrm{H}), 8.15 \sim 8.04(\mathrm{~m}, 2 \mathrm{H}), 7.95(\mathrm{~d}, J=9.2 \mathrm{~Hz}, 1 \mathrm{H}), 7.57(\mathrm{~d}$, $J=9.3 \mathrm{~Hz}, 1 \mathrm{H}), 7.42 \sim 7.31(\mathrm{~m}, 4 \mathrm{H}), 7.08 \sim 7.02(\mathrm{~m}, 2 \mathrm{H})$; ${ }^{13} \mathrm{C}$ NMR (101 MHz, Chloroform- $d$ ) $\delta:$ 142.6, 131.6, $131.5,131.3,131.2,131.1,128.9,128.8,128.4,127.3$, $126.7,126.6,126.2,125.9,125.9,125.7,123.5,124.8$, 122.8, 120.5, 120.0, 110.4; ESI-HRMS calcd for $\mathrm{C}_{28} \mathrm{H}_{17} \mathrm{~N}$ 367.1361 , found 367.1360 .

3,6-二氟-9-(1-萠基)-咔唑(5ab): 白色固体，产率 
$62 \%$. m.p. $206 \sim 207{ }^{\circ} \mathrm{C} ;{ }^{1} \mathrm{H}$ NMR $(400 \mathrm{MHz}$, Chloroform-d) $\delta: 8.35(\mathrm{~d}, J=8.2 \mathrm{~Hz}, 1 \mathrm{H}), 8.29(\mathrm{~d}, J=7.7 \mathrm{~Hz}$, $1 \mathrm{H}), 8.25 \sim 8.17(\mathrm{~m}, 3 \mathrm{H}), 8.10 \sim 8.02(\mathrm{~m}, 2 \mathrm{H}), 7.96$ (d, $J=$ $9.2 \mathrm{~Hz}, 1 \mathrm{H}), 7.85$ (dd, $J=8.8,2.6 \mathrm{~Hz}, 2 \mathrm{H}), 7.49$ (d, $J=9.3$ $\mathrm{Hz}, 1 \mathrm{H}), 7.10$ (td, $J=9.0,2.6 \mathrm{~Hz}, 2 \mathrm{H}), 6.94$ (dd, $J=8.9$, $4.3 \mathrm{~Hz}, 2 \mathrm{H}) ;{ }^{13} \mathrm{C}$ NMR (101 MHz, Chloroform- $d$ ) $\delta: 157.8$ (d, $J=237.0 \mathrm{~Hz}$ ), 139.8, 131.7, 131.2, 131.0, 130.6, 129.0, $128.8,128.6,127.2,126.7,126.5,126.1,126.0,125.9$, 125.7, 124.7, 123.4 (dd, $J=9.5,4.0 \mathrm{~Hz}), 122.3,114.6$ (d, $J=25.5 \mathrm{~Hz}), 111.3(\mathrm{~d}, J=9.3 \mathrm{~Hz}), 106.3(\mathrm{~d}, J=24.1 \mathrm{~Hz})$; ESI-HRMS calcd for $\mathrm{C}_{28} \mathrm{H}_{15} \mathrm{~F}_{2} \mathrm{~N}$ 403.1173, found 403.1168.

3,6-二氯-9-(1-萠基)-咔唑(5ac): 白色固体, 产率 65\%. m.p. $224 \sim 225{ }^{\circ} \mathrm{C}$; ${ }^{1} \mathrm{H}$ NMR $(400 \mathrm{MHz}$, Chloroform- $d$ ) $\delta: 8.36(\mathrm{dd}, J=8.2,1.6 \mathrm{~Hz}, 1 \mathrm{H}), 8.31(\mathrm{~d}, J=7.6$ $\mathrm{Hz}, 1 \mathrm{H}), 8.22(\mathrm{dt}, J=7.5,3.0 \mathrm{~Hz}, 3 \mathrm{H}), 8.16(\mathrm{~d}, J=2.0 \mathrm{~Hz}$, 2H), 8.07 (ddd, $J=17.9,7.9,1.6 \mathrm{~Hz}, 2 \mathrm{H}), 7.97$ (dd, $J=$ 9.2, $1.4 \mathrm{~Hz}, 1 \mathrm{H}), 7.43(\mathrm{dd}, J=9.2,1.6 \mathrm{~Hz}, 1 \mathrm{H}), 7.32$ (dd, $J=8.8,2.0 \mathrm{~Hz}, 2 \mathrm{H}), 6.94(\mathrm{dd}, J=8.7,1.5 \mathrm{~Hz}, 2 \mathrm{H}) ;{ }^{13} \mathrm{C}$ NMR (101 MHz, Chloroform- $d$ ) $\delta$ : 141.4, 131.9, 131.3, $131.0,130.0,129.2,128.7,128.7,127.3,127.0,126.8$, $126.4,126.3,126.1,126.0,125.9,125.7,124.6,123.6$, 122.1, 120.4, 111.7; ESI-HRMS calcd for $\mathrm{C}_{28} \mathrm{H}_{15} \mathrm{Cl}_{2} \mathrm{~N}$ 435.0582, found 435.0582 .

3,6-二澳-9-(1-萠基)-咔唑 $(\mathbf{5 a d})$ : 白色固体, 产率 $65 \%$. m.p. $213 \sim 214{ }^{\circ} \mathrm{C} ;{ }^{1} \mathrm{H}$ NMR $(400 \mathrm{MHz}$, Chloroform- $d) \delta: 8.38 \sim 8.28(\mathrm{~m}, 4 \mathrm{H}), 8.26 \sim 8.17(\mathrm{~m}, 3 \mathrm{H})$, $8.12 \sim 8.01(\mathrm{~m}, 2 \mathrm{H}), 7.96(\mathrm{dd}, J=9.2,1.4 \mathrm{~Hz}, 1 \mathrm{H}), 7.48 \sim$ 7.37 (m, 3H), 6.89 (dd, $J=8.7,1.4 \mathrm{~Hz}, 2 \mathrm{H}) ;{ }^{13} \mathrm{C}$ NMR (101 MHz, Chloroform- $d$ ) $\delta: 141.5,131.9,131.2,131.0$, $129.9,129.7,129.2,128.8,128.6,127.2,126.8,126.3$, 126.3, 126.1, 125.9, 125.7, 124.6, 124.0, 123.5, 122.0, 113.3, 112.1; ESI-HRMS calcd for $\mathrm{C}_{28} \mathrm{H}_{15} \mathrm{Br}_{2} \mathrm{~N}$ 524.9551 , found 524.9553 .

3,6-二碘-9-(1-萠基)-咔唑(5ae): 白色固体, 产率 43\%. m.p. 256 257 ${ }^{\circ} \mathrm{C} ;{ }^{1} \mathrm{H}$ NMR (400 MHz, Chloroform- $d$ ) $\delta: 8.52(\mathrm{~s}, 2 \mathrm{H}), 8.35(\mathrm{~d}, J=8.0 \mathrm{~Hz}, 1 \mathrm{H}), 8.30(\mathrm{~d}$, $J=7.5 \mathrm{~Hz}, 1 \mathrm{H}), 8.25 \sim 8.16(\mathrm{~m}, 3 \mathrm{H}), 8.08$ (td, $J=7.9,1.3$ $\mathrm{Hz}, 1 \mathrm{H}), 8.02(\mathrm{dd}, J=8.0,1.5 \mathrm{~Hz}, 1 \mathrm{H}), 7.97 \sim 7.92(\mathrm{~m}$, $1 \mathrm{H}), 7.60(\mathrm{~d}, J=8.7 \mathrm{~Hz}, 2 \mathrm{H}), 7.40(\mathrm{dd}, J=9.2,1.4 \mathrm{~Hz}$, $1 \mathrm{H}), 6.82 \sim 6.76(\mathrm{~m}, 2 \mathrm{H}) ;{ }^{13} \mathrm{C}$ NMR $(101 \mathrm{MHz}$, Chloroform- $d$ ) $\delta: 141.7,135.2,131.9,131.2,131.0,129.7,129.6$, $129.2,128.8,128.6,127.2,126.8,126.3,126.3,126.1$, 125.9, 125.7, 124.6, 124.5, 122.0, 112.6, 83.2; ESI-HRMS calcd for $\mathrm{C}_{28} \mathrm{H}_{15} \mathrm{I}_{2} \mathrm{~N} \quad$ 618.9294, found 618.9297. 2,7-二溴-9-(1-萠基)-咔唑(5af)：白色固体，产率 55\%. m.p. 208 209 ${ }^{\circ} \mathrm{C}$; ${ }^{1} \mathrm{H}$ NMR (400 MHz, Chloroform- $d$ ) $\delta: 8.38$ (dd, $J=8.1,2.5 \mathrm{~Hz}, 1 \mathrm{H}), 8.32(\mathrm{~d}, J=7.6$ $\mathrm{Hz}, 1 \mathrm{H}), 8.22(\mathrm{dd}, J=8.2,5.5 \mathrm{~Hz}, 3 \mathrm{H}), 8.12 \sim 7.98(\mathrm{~m}$, $5 \mathrm{H}), 7.48 \sim 7.43(\mathrm{~m}, 3 \mathrm{H}), 7.15(\mathrm{~d}, J=1.5 \mathrm{~Hz}, 2 \mathrm{H}) ;{ }^{13} \mathrm{C}$ NMR (101 MHz, Chloroform- $d$ ) $\delta$ : 143.5, 132.1, 131.2, $131.0,129.5,128.9,128.7,127.2,126.8,126.5,126.3$, $126.2,125.9,125.8,125.1,124.6,123.8,121.9,121.8$, 121.7, 120.3, 113.6; ESI-HRMS calcd for $\mathrm{C}_{28} \mathrm{H}_{15} \mathrm{Br}_{2} \mathrm{~N}$ 524.9551, found 524.9548 .

3,6-二溴-9-(1-萗基)-咔唑 $(5 \mathbf{b})$ : 白色固体, 产率 $40 \%$. m.p. $165 \sim 166{ }^{\circ} \mathrm{C} ;{ }^{1} \mathrm{H}$ NMR $(400 \mathrm{MHz}$, Chloroform- $d$ ) $\delta: 8.27(\mathrm{~d}, J=1.9 \mathrm{~Hz}, 2 \mathrm{H}), 8.07(\mathrm{~d}, J=8.2 \mathrm{~Hz}$, $1 \mathrm{H}), 8.02(\mathrm{~d}, J=8.1 \mathrm{~Hz}, 1 \mathrm{H}), 7.72 \sim 7.65(\mathrm{~m}, 1 \mathrm{H}), 7.62 \sim$ $7.52(\mathrm{~m}, 2 \mathrm{H}), 7.44$ (dd, $J=8.7,1.9 \mathrm{~Hz}, 2 \mathrm{H}), 7.35$ (ddd, $J=$ 8.2, 6.9, $1.1 \mathrm{~Hz}, 1 \mathrm{H}), 7.14(\mathrm{~d}, J=8.5 \mathrm{~Hz}, 1 \mathrm{H}), 6.87(\mathrm{~d}, J=$ $8.7 \mathrm{~Hz}, 2 \mathrm{H}) ;{ }^{13} \mathrm{C}$ NMR (101 MHz, Chloroform-d) $\delta: 141.2$, $135.0,133.1,130.7,129.7,129.6,128.8,127.5,127.1$, $126.8,126.0,123.9,123.4,123.0,113.2,112.1$; ESIHRMS calcd for $\mathrm{C}_{22} \mathrm{H}_{13} \mathrm{Br}_{2} \mathrm{~N} 450.9394$, found 450.9395 .

3,6-二澳-9-(2-甲氧基-1-荎基)-咔唑(5c)：淡黄色固 体, 产率 48\%. m.p. 164 165 ${ }^{\circ} \mathrm{C} ;{ }^{1} \mathrm{H}$ NMR (400 MHz, Chloroform- $d$ ) $\delta: 8.27$ (d, $J=1.8 \mathrm{~Hz}, 2 \mathrm{H}), 8.08$ (d, $J=9.0$ $\mathrm{Hz}, 1 \mathrm{H}), 7.94(\mathrm{~d}, J=8.2 \mathrm{~Hz}, 1 \mathrm{H}), 7.49$ (d, $J=8.9 \mathrm{~Hz}, 1 \mathrm{H})$, $7.45 \sim 7.38(\mathrm{~m}, 3 \mathrm{H}), 7.31(\mathrm{dd}, J=8.5,6.8 \mathrm{~Hz}, 1 \mathrm{H}), 7.01(\mathrm{~d}$, $J=8.7 \mathrm{~Hz}, 1 \mathrm{H}), 6.77(\mathrm{~d}, J=8.7 \mathrm{~Hz}, 2 \mathrm{H}), 3.76(\mathrm{~d}, J=1.5$ $\mathrm{Hz}, 3 \mathrm{H}) ;{ }^{13} \mathrm{C}$ NMR (101 MHz, Chloroform- $d$ ) $\delta: 154.5$, $140.7,132.2,131.1,129.5,129.4,128.5,128.0,124.6$, 124.0, 123.3, 122.1, 117.4, 114.0, 112.9, 112.1, 56.7; ESIHRMS calcd for $\mathrm{C}_{23} \mathrm{H}_{15} \mathrm{Br}_{2} \mathrm{NO} 480.9500$, found 480.9498 .

3,6-二溴-9-(2-甲硫基-1-菜基)-咔唑(5d): 白色固体, 产率 34\%. m.p. $180 \sim 181{ }^{\circ} \mathrm{C} ;{ }^{1} \mathrm{H}$ NMR (400 MHz, Chloroform- $d$ ) $\delta: 8.29(\mathrm{~d}, J=2.0 \mathrm{~Hz}, 2 \mathrm{H}), 8.07(\mathrm{~d}, J=9.0 \mathrm{~Hz}$, $1 \mathrm{H}), 7.96(\mathrm{~d}, J=8.1 \mathrm{~Hz}, 1 \mathrm{H}), 7.60(\mathrm{~d}, J=8.8 \mathrm{~Hz}, 1 \mathrm{H})$, $7.49 \sim 7.41(\mathrm{~m}, 3 \mathrm{H}), 7.30(\mathrm{t}, J=7.6 \mathrm{~Hz}, 1 \mathrm{H}), 6.85(\mathrm{~d}, J=$ $8.2 \mathrm{~Hz}, 1 \mathrm{H}), 6.74(\mathrm{~d}, J=8.7 \mathrm{~Hz}, 2 \mathrm{H}), 2.40(\mathrm{~s}, 3 \mathrm{H}) ;{ }^{13} \mathrm{C}$ NMR (101 MHz, Chloroform- $d$ ) $\delta: 139.8,138.2,132.1$, $131.6,130.3,129.6,128.5,128.3,127.5,126.1,124.0$, 123.6, 123.0, 121.9, 113.3, 112.0, 14.9; ESI-HRMS calcd for $\mathrm{C}_{23} \mathrm{H}_{15} \mathrm{Br}_{2} \mathrm{NS} \quad$ 496.9271, found 496.9266.

3,6-二澳-9-(2,7-二甲氧基-1-荎基)-咔唑(5e)：黄色 固体, 产率 50\%. m.p. $157 \sim 158{ }^{\circ} \mathrm{C} ;{ }^{1} \mathrm{H}$ NMR $(400 \mathrm{MHz}$, Chloroform- $d$ ) $\delta: 8.25$ (d, $J=1.8 \mathrm{~Hz}, 2 \mathrm{H}), 7.97$ (d, $J=9.1$ $\mathrm{Hz}, 1 \mathrm{H}), 7.82(\mathrm{~d}, J=9.0 \mathrm{~Hz}, 1 \mathrm{H}), 7.43(\mathrm{dd}, J=8.6,1.9 \mathrm{~Hz}$, $2 \mathrm{H}), 7.30(\mathrm{~d}, J=9.0 \mathrm{~Hz}, 1 \mathrm{H}), 7.05(\mathrm{dd}, J=9.0,2.5 \mathrm{~Hz}$, 
1H), 6.79 (d, $J=8.7 \mathrm{~Hz}, 2 \mathrm{H}), 6.26$ (d, $J=2.5 \mathrm{~Hz}, 1 \mathrm{H}), 3.73$ (s, 3H), 3.46 (s, 3H); ${ }^{13} \mathrm{C}$ NMR (101 MHz, Chloroform-d) $\delta$ : 159.6, 155.1, 140.5, 133.7, 130.7, 130.2, 129.3, 124.9, 124.0, 123.3, 117.4, 116.5, 112.8, 112.2, 111.0, 100.3, 56.5, 55.3; ESI-HRMS calcd for $\mathrm{C}_{24} \mathrm{H}_{17} \mathrm{Br}_{2} \mathrm{NO}_{2} 510.9606$, found 510.9602 .

3,6-二溴-9-(6-溴-2-二甲氧基-1-菜基)-咔唑(5f)：白 色固体, 产率 69\%. m.p. 206 207 ${ }^{\circ} \mathrm{C} ;{ }^{1} \mathrm{H}$ NMR (400 MHz, Chloroform- $d$ ) $\delta: 8.27$ (d, $J=1.8 \mathrm{~Hz}, 2 \mathrm{H}), 8.10$ (d, $J=2.0 \mathrm{~Hz}, 1 \mathrm{H}), 7.99$ (d, $J=9.1 \mathrm{~Hz}, 1 \mathrm{H}), 7.51$ (d, $J=9.1$ $\mathrm{Hz}, 1 \mathrm{H}), 7.43$ (dd, $J=8.7,1.9 \mathrm{~Hz}, 2 \mathrm{H}), 7.35$ (dd, $J=8.9$, $1.9 \mathrm{~Hz}, 1 \mathrm{H}), 6.90(\mathrm{~d}, J=9.1 \mathrm{~Hz}, 1 \mathrm{H}), 6.74$ (d, $J=8.7 \mathrm{~Hz}$, 2H), $3.76(\mathrm{~s}, 3 \mathrm{H}) ;{ }^{13} \mathrm{C}$ NMR (101 MHz, Chloroform- $d$ ) $\delta$ : $154.7,140.5,131.3,130.7,130.4,130.1,129.5,124.0$, 124.0, 123.4, 118.4, 117.6, 115.0, 113.1, 111.9, 56.6; ESI-HRMS calcd for $\mathrm{C}_{23} \mathrm{H}_{14} \mathrm{Br}_{3} \mathrm{NO}$ 558.8605, found: 558.8603 .

3,6-二溴-9-(5-范基)-咔唑(5g)：淡黄色固体，产率 61\%. m.p. 153 $154{ }^{\circ} \mathrm{C}$; ${ }^{1} \mathrm{H}$ NMR (400 MHz, Chloroform- $d$ ) $\delta: 8.27(\mathrm{~d}, J=1.9 \mathrm{~Hz}, 2 \mathrm{H}), 7.54(\mathrm{~d}, J=7.3 \mathrm{~Hz}$, $1 \mathrm{H}), 7.48 \sim 7.42(\mathrm{~m}, 3 \mathrm{H}), 7.39 \sim 7.34(\mathrm{~m}, 2 \mathrm{H}), 7.32(\mathrm{~d}, J=$ $7.3 \mathrm{~Hz}, 1 \mathrm{H}), 6.95$ (d, $J=8.7 \mathrm{~Hz}, 2 \mathrm{H}), 6.91$ (d, $J=7.9 \mathrm{~Hz}$, 1H), 3.54 (s, 4H); ${ }^{13} \mathrm{C}$ NMR (101 MHz, Chloroform-d) $\delta$ : $147.8,146.8,141.0,140.8,129.4,129.1,128.8,128.7$, $128.1,123.8,123.3,120.5,119.5,118.5,112.9,112.1$, 31.0, 30.5; ESI-HRMS calcd for $\mathrm{C}_{24} \mathrm{H}_{15} \mathrm{Br}_{2} \mathrm{~N} \quad 476.9551$, found 476.9554 .

3,6-二溴-9-(9-菲基)-咔唑(5h)：白色固体，产率 59\%. m.p. 160 161 ${ }^{\circ} \mathrm{C}$; ${ }^{1} \mathrm{H}$ NMR (400 MHz, Chloroform- $d$ ) $\delta: 8.83(\mathrm{t}, J=9.0 \mathrm{~Hz}, 2 \mathrm{H}), 8.29(\mathrm{~d}, J=2.1 \mathrm{~Hz}$, 2H), 7.94 (d, $J=8.8 \mathrm{~Hz}, 2 \mathrm{H}), 7.81$ (t, $J=7.7 \mathrm{~Hz}, 1 \mathrm{H}), 7.71$ $(\mathrm{q}, J=7.3 \mathrm{~Hz}, 2 \mathrm{H}), 7.47 \sim 7.38(\mathrm{~m}, 3 \mathrm{H}), 7.15(\mathrm{~d}, J=8.3$ $\mathrm{Hz}, 1 \mathrm{H}), 6.94$ (d, $J=8.7 \mathrm{~Hz}, 2 \mathrm{H}) ;{ }^{13} \mathrm{C}$ NMR $(101 \mathrm{MHz}$, Chloroform- $d$ ) $\delta$ : 141.2, 131.9, 131.7, 131.5, 130.8, 129.6, 129.2 , 129.1, 128.1, 128.0, 127.8, 127.6, 127.5, 123.9, 123.8, 123.5, 123.4, 123.0, 113.2, 112.1; ESI-HRMS calcd for $\mathrm{C}_{26} \mathrm{H}_{15} \mathrm{Br}_{2} \mathrm{~N}$ 500.9551, found 500.9548.

3,6-二溴-9-(10-溴-9-菲基)-咔唑(5i): 白色固体，产 率 37\%. m.p. 181 $182{ }^{\circ} \mathrm{C}$; ${ }^{1} \mathrm{H}$ NMR (400 MHz, Chloroform- $d$ ) $\delta: 8.83(\mathrm{t}, J=9.2 \mathrm{~Hz}, 2 \mathrm{H}), 8.58 \sim 8.49(\mathrm{~m}, 1 \mathrm{H})$, $8.32(\mathrm{~d}, J=2.3 \mathrm{~Hz}, 2 \mathrm{H}), 7.93 \sim 7.68(\mathrm{~m}, 3 \mathrm{H}), 7.49 \sim 7.34$ (m, 3H), 7.00 (d, $J=8.4 \mathrm{~Hz}, 1 \mathrm{H}), 6.80(\mathrm{dd}, J=9.0,3.2 \mathrm{~Hz}$, $2 \mathrm{H}) ;{ }^{13} \mathrm{C}$ NMR (101 MHz, Chloroform-d) $\delta: 139.9,131.7$, $131.2,130.9,130.5,130.0,129.8,129.6,129.0,128.4$, $128.4,128.3,126.1,124.2,124.0,123.7,123.5,123.1$,
113.6, 112.1; ESI-HRMS calcd for $\mathrm{C}_{26} \mathrm{H}_{14} \mathrm{Br}_{3} \mathrm{~N}$ 578.8656, found 578.8656 .

3,6-二溴-9-(9-蒽基)-咔唑 $(\mathbf{5 j})$ ：白色固体，产率 38\%. m.p. 209 $210{ }^{\circ} \mathrm{C}$; ${ }^{1} \mathrm{H}$ NMR (400 MHz, Chloroform- $d) \delta: 8.73(\mathrm{~d}, J=3.9 \mathrm{~Hz}, 1 \mathrm{H}), 8.36(\mathrm{~d}, J=2.8 \mathrm{~Hz}$, $2 \mathrm{H}), 8.18(\mathrm{dd}, J=9.6,3.5 \mathrm{~Hz}, 2 \mathrm{H}), 7.53(\mathrm{td}, J=7.4,6.9$, $3.5 \mathrm{~Hz}, 2 \mathrm{H}), 7.42 \sim 7.29(\mathrm{~m}, 4 \mathrm{H}), 7.21 \sim 7.13(\mathrm{~m}, 2 \mathrm{H}), 6.62$ $(\mathrm{dt}, J=8.7,2.8 \mathrm{~Hz}, 2 \mathrm{H}) ;{ }^{13} \mathrm{C}$ NMR (101 MHz, Chloroform-d) $\delta$ : 141.6, 132.1, 129.8, 129.0, 127.6, 126.1, 123.9, 123.5, 123.0, 113.3, 112.2; ESI-HRMS calcd for $\mathrm{C}_{26} \mathrm{H}_{15} \mathrm{Br}_{2} \mathrm{~N} \quad$ 500.9551, found 500.9554 .

3,6-二溴-9-(10-苯基-9-葸基)-咔唑(5k)：白色固体， 产率 62\%. m.p. 217 218 ${ }^{\circ} \mathrm{C}$; ${ }^{1} \mathrm{H}$ NMR (400 MHz, Chloroform-d) $\delta: 8.39(\mathrm{~s}, 2 \mathrm{H}), 7.84(\mathrm{~d}, J=8.7 \mathrm{~Hz}, 2 \mathrm{H}), 7.65$ $(\mathrm{dd}, J=13.1,7.0 \mathrm{~Hz}, 3 \mathrm{H}), 7.57$ (d, $J=7.1 \mathrm{~Hz}, 2 \mathrm{H}), 7.44 \sim$ $7.36(\mathrm{~m}, 4 \mathrm{H}), 7.31(\mathrm{t}, J=7.6 \mathrm{~Hz}, 2 \mathrm{H}), 7.22(\mathrm{~d}, J=8.7 \mathrm{~Hz}$, $2 \mathrm{H}), 6.73(\mathrm{~d}, J=8.7 \mathrm{~Hz}, 2 \mathrm{H}) ;{ }^{13} \mathrm{C}$ NMR $(101 \mathrm{MHz}$, Chloroform- $d$ ) $\delta: 141.7,139.8,138.2,131.2,130.8,129.8$, $129.5,128.7,128.1,127.9,127.4,127.3,125.9,123.9$, 123.6, 122.9, 113.3, 112.3; ESI-HRMS calcd for $\mathrm{C}_{26} \mathrm{H}_{15} \mathrm{Br}_{2} \mathrm{~N}$ 576.9864, found 576.9870.

3,6-二溴-9-(3-苂葱基)-咔唑(5l): 黄色固体，产率 50\%. m.p. 214 $215{ }^{\circ} \mathrm{C}$; ${ }^{1} \mathrm{H}$ NMR (400 MHz, Chloroform-d) $\delta: 8.26(\mathrm{~d}, J=2.4 \mathrm{~Hz}, 2 \mathrm{H}), 8.06(\mathrm{dd}, J=7.1,2.4$ $\mathrm{Hz}, 1 \mathrm{H}), 7.95$ (qd, $J=5.7,2.6 \mathrm{~Hz}, 3 \mathrm{H}), 7.69$ (dd, $J=7.2$, $2.3 \mathrm{~Hz}, 1 \mathrm{H}), 7.51 \sim 7.40(\mathrm{~m}, 5 \mathrm{H}), 7.22(\mathrm{dd}, J=8.2,2.2 \mathrm{~Hz}$, $1 \mathrm{H}), 7.03(\mathrm{dd}, J=8.7,2.3 \mathrm{~Hz}, 2 \mathrm{H}) ;{ }^{13} \mathrm{C}$ NMR $(101 \mathrm{MHz}$, Chloroform- $d$ ) $\delta$ : 141.4, 139.9, 138.8, 138.2, 137.8, 134.0, $133.0,129.6,129.2,128.4,128.3,128.2,127.5,124.0$, $123.3,123.1,122.0,122.0,121.1,120.4,113.3,112.1$; ESI-HRMS calcd for $\mathrm{C}_{28} \mathrm{H}_{15} \mathrm{Br}_{2} \mathrm{~N}$ 524.9551, found 524.9556

3,6-二溴-9-(2-三亚苯基)-咔唑(5m)：白色固体，产 率 22\%. m.p. 194 $195{ }^{\circ} \mathrm{C} ;{ }^{1} \mathrm{H}$ NMR (400 MHz, Chloroform- $d$ ) $\delta: 8.87(\mathrm{~d}, J=8.0 \mathrm{~Hz}, 1 \mathrm{H}), 8.71(\mathrm{dt}, J=6.0,3.2$ $\mathrm{Hz}, 1 \mathrm{H}), 8.63(\mathrm{dt}, J=7.3,3.5 \mathrm{~Hz}, 1 \mathrm{H}), 8.54(\mathrm{~d}, J=8.1 \mathrm{~Hz}$, 1H), $8.27(\mathrm{~d}, J=1.9 \mathrm{~Hz}, 2 \mathrm{H}), 7.79$ (t, $J=7.8 \mathrm{~Hz}, 1 \mathrm{H}), 7.73$ $(\mathrm{dt}, J=6.3,3.5 \mathrm{~Hz}, 2 \mathrm{H}), 7.58(\mathrm{~d}, J=7.5 \mathrm{~Hz}, 1 \mathrm{H}), 7.43 \sim$ $7.34(\mathrm{~m}, 3 \mathrm{H}), 7.01(\mathrm{~d}, J=8.6 \mathrm{~Hz}, 1 \mathrm{H}), 6.92(\mathrm{~d}, J=8.7 \mathrm{~Hz}$, $2 \mathrm{H}), 6.82(\mathrm{dd}, J=8.4,6.7 \mathrm{~Hz}, 1 \mathrm{H}) ;{ }^{13} \mathrm{C} \mathrm{NMR}(101 \mathrm{MHz}$, Chloroform- $d$ ) $\delta$ : 139.4, 133.5, 133.2, 130.7, 130.5, 129.8, $129.7,129.6,128.2,127.9,127.8,127.7,127.3,125.5$, 124.5, 124.4, 123.9, 123.6, 123.4, 123.4, 113.5, 112.3; ESI-HRMS calcd for $\mathrm{C}_{30} \mathrm{H}_{17} \mathrm{Br}_{2} \mathrm{~N}$ 550.9707, found 550.9707. 
3,6-二溴-9-(3,4-二甲氧基苯基)-咔唑(5n): 白色固 体, 产率 14\%. m.p. 198 $199{ }^{\circ} \mathrm{C} ;{ }^{1} \mathrm{H}$ NMR $(400 \mathrm{MHz}$, Chloroform- $d$ ) $\delta: 8.19$ (d, $J=2.0 \mathrm{~Hz}, 2 \mathrm{H}), 7.50$ (dd, $J=$ $8.6,2.0 \mathrm{~Hz}, 2 \mathrm{H}), 7.20(\mathrm{~d}, J=8.8 \mathrm{~Hz}, 2 \mathrm{H}), 7.08 \sim 7.01(\mathrm{~m}$, $2 \mathrm{H}), 6.94(\mathrm{~d}, J=1.9 \mathrm{~Hz}, 1 \mathrm{H}), 4.00$ (s, 3H), 3.88 (s, 3H); ${ }^{13} \mathrm{C}$ NMR (101 MHz, Chloroform- $d$ ) $\delta$ : 150.2, 149.1, $140.5,129.6,129.5,123.8,123.3,119.7,113.0,111.9$, 111.6, 110.6, 56.3, 56.3; ESI-HRMS calcd for $\mathrm{C}_{20} \mathrm{H}_{15} \mathrm{Br}_{2} \mathrm{NO}_{2} \quad 460.9449$, found 460.9446 .

3,6-二溴-9-(2,4,6-三甲氧基苯基)-咔唑(5o): 白色固 体, 产率 19\%. m.p. 212 213 ${ }^{\circ} \mathrm{C} ;{ }^{1} \mathrm{H}$ NMR $(400 \mathrm{MHz}$, Chloroform- $d$ ) $\delta: 8.17$ (d, $J=1.9 \mathrm{~Hz}, 2 \mathrm{H}), 7.45$ (dd, $J=$ 8.7, $1.9 \mathrm{~Hz}, 2 \mathrm{H}), 6.89$ (d, $J=8.7 \mathrm{~Hz}, 2 \mathrm{H}), 6.30(\mathrm{~s}, 2 \mathrm{H})$, 3.93 (s, 3H), 3.62 (s, 9H); ${ }^{13} \mathrm{C}$ NMR (101 MHz, Chloroform-d) $\delta: 161.7,158.3,140.6,129.0,123.8,123.1,112.4$, 111.9, 106.0, 91.3, 56.0, 55.8; ESI-HRMS calcd for $\mathrm{C}_{21} \mathrm{H}_{17} \mathrm{Br}_{2} \mathrm{NO}_{3} \quad 490.9555$, found 490.9558 .

4-(9-咔唑基)- $N, N$-二苯基苯胺 (5pa): 白色固体, 产 率 $61 \%$. m.p. $176 \sim 177{ }^{\circ} \mathrm{C}$ (文献值 ${ }^{[13]}$ : m.p. $172 \sim$ $\left.174{ }^{\circ} \mathrm{C}\right) ;{ }^{1} \mathrm{H}$ NMR (400 MHz, Chloroform- $d$ ) $\delta: 8.17$ (d, $J$ $=7.7 \mathrm{~Hz}, 2 \mathrm{H}), 7.49 \sim 7.39(\mathrm{~m}, 5 \mathrm{H}), 7.38 \sim 7.21(\mathrm{~m}, 13 \mathrm{H})$, $7.11(\mathrm{t}, J=7.4 \mathrm{~Hz}, 2 \mathrm{H}) ;{ }^{13} \mathrm{C}$ NMR $(101 \mathrm{MHz}$, Chloroform-d) $\delta: 147.6,147.2,141.2,131.5,129.6,128.0,126.0$, $124.9, \quad 124.1, \quad 123.5, \quad 123.3, \quad 120.4, \quad 119.8, \quad 110.0$; ESI-HRMS calcd for $\mathrm{C}_{30} \mathrm{H}_{22} \mathrm{~N}_{2}$ 410.1783, found 410.1786.

4-(3,6-二氯-9-咔唑基)- $N, N$-二苯基苯胺 $(5 \mathbf{p b})$ : 白色 固体, 产率 44\%. m.p. $214 \sim 215{ }^{\circ} \mathrm{C} ;{ }^{1} \mathrm{H}$ NMR (400 MHz, Chloroform- $d$ ) $\delta: 8.03$ (d, $J=1.9 \mathrm{~Hz}, 2 \mathrm{H}), 7.41 \sim 7.29$ (m, $10 \mathrm{H}), 7.27 \sim 7.20(\mathrm{~m}, 6 \mathrm{H}), 7.12(\mathrm{t}, J=7.4 \mathrm{~Hz}, 2 \mathrm{H}) ;{ }^{13} \mathrm{C}$ NMR (101 MHz, Chloroform- $d$ ) $\delta$ : 147.8, 147.5, 140.0, $130.3,129.7,127.8,126.8,125.6,125.1,123.8,123.7$, 123.4, 120.2, 111.3; ESI-HRMS calcd for $\mathrm{C}_{30} \mathrm{H}_{20} \mathrm{Cl}_{2} \mathrm{~N}_{2}$ 478.1004, found 478.1005 .

4-(3,6-二澳-9-咔唑基)- $\mathrm{N}, \mathrm{N}$-二苯基苯胺 (5pc): 棕色 固体, 产率 48\%. m.p. $225 \sim 226{ }^{\circ} \mathrm{C} ;{ }^{1} \mathrm{H}$ NMR (400 MHz, Chloroform- $d$ ) $\delta: 8.18(\mathrm{~d}, J=1.9 \mathrm{~Hz}, 2 \mathrm{H}), 7.50$ (dd, $J=$ 8.7, $1.9 \mathrm{~Hz}, 2 \mathrm{H}), 7.36 \sim 7.18(\mathrm{~m}, 15 \mathrm{H}), 7.10(\mathrm{t}, J=7.3 \mathrm{~Hz}$, $2 \mathrm{H}) ;{ }^{13} \mathrm{C}$ NMR (101 MHz, Chloroform- $d$ ) $\delta: 147.8,147.4$, $140.2,130.1,129.7,129.4,127.8,125.1,123.9,123.9$, 123.6, 123.3, 113.0, 111.7; ESI-HRMS calcd for $\mathrm{C}_{30} \mathrm{H}_{20^{-}}$ $\mathrm{Br}_{2} \mathrm{~N}_{2}$ 567.9973, found 567.9978.

4-(2,7-二溴-9-咔唑基)- $N, N$-二苯基苯胺 (5pd): 白色 固体, 产率 11\%. m.p. $168 \sim 169{ }^{\circ} \mathrm{C} ;{ }^{1} \mathrm{H}$ NMR $(400 \mathrm{MHz}$, Chloroform- $d$ ) $\delta: 7.93$ (d, $J=8.3 \mathrm{~Hz}, 2 \mathrm{H}), 7.52$ (d, $J=1.8$
$\mathrm{Hz}, 2 \mathrm{H}), 7.42 \sim 7.33(\mathrm{~m}, 6 \mathrm{H}), 7.29(\mathrm{~d}, J=8.9 \mathrm{~Hz}, 2 \mathrm{H})$, $7.26 \sim 7.22(\mathrm{~m}, 6 \mathrm{H}), 7.12(\mathrm{t}, J=7.6 \mathrm{~Hz}, 2 \mathrm{H}) ;{ }^{13} \mathrm{C}$ NMR (101 MHz, Chloroform-d) $\delta: 148.1,147.4,142.3,129.7$, $129.6,128.0,125.3,123.9,123.6,123.5,121.7,121.6$, 120.0, 113.2; ESI-HRMS calcd for $\mathrm{C}_{30} \mathrm{H}_{20} \mathrm{Br}_{2} \mathrm{~N}_{2}$ 567.9973 , found 567.9976 .

4-溴- $N$-(4-(3,6-二溴-9-咔唑基)苯基- $N$-苯基苯胺 (5q)：白色固体，产率 48\%. m.p. $198 \sim 199{ }^{\circ} \mathrm{C} ;{ }^{1} \mathrm{H}$ NMR (400 MHz, Chloroform- $d$ ) $\delta: 8.18$ (d, $J=2.0 \mathrm{~Hz}, 2 \mathrm{H}), 7.51$ (dd, $J=8.8,1.9 \mathrm{~Hz}, 2 \mathrm{H}), 7.45 \sim 7.39(\mathrm{~m}, 2 \mathrm{H}), 7.38 \sim 7.17$ (m, 10H), $7.14(\mathrm{t}, J=7.4 \mathrm{~Hz}, 1 \mathrm{H}), 7.11 \sim 7.04(\mathrm{~m}, 2 \mathrm{H}) ;{ }^{13} \mathrm{C}$ NMR (101 MHz, Chloroform- $d$ ) $\delta: 147.3,147.0,146.6$, $140.1,132.7,130.7,129.9,129.4,127.9,126.1,125.3$, 124.3, 124.1, 123.9, 123.3, 116.1, 113.1, 111.7; ESI-HRMS calcd for $\mathrm{C}_{30} \mathrm{H}_{19} \mathrm{Br}_{3} \mathrm{~N}_{2} \quad 645.9078$, found 645.9073 .

3',6'-二溴-9-乙基-3,9'-二咔唑(5r): 白色固体, 产率 37\%. m.p. $168 \sim 169{ }^{\circ} \mathrm{C} ;{ }^{1} \mathrm{H}$ NMR (400 MHz, Chloroform-d) $\delta: 8.23(\mathrm{~d}, J=1.9 \mathrm{~Hz}, 2 \mathrm{H}), 8.17(\mathrm{~d}, J=1.9 \mathrm{~Hz}$, $1 \mathrm{H}), 8.09 \sim 8.05(\mathrm{~m}, 1 \mathrm{H}), 7.62 \sim 7.47(\mathrm{~m}, 6 \mathrm{H}), 7.28(\mathrm{~d}, J=$ $7.0 \mathrm{~Hz}, 1 \mathrm{H}), 7.22(\mathrm{~d}, J=8.7 \mathrm{~Hz}, 2 \mathrm{H}), 4.48(\mathrm{q}, J=7.2 \mathrm{~Hz}$, $2 \mathrm{H}), 1.53(\mathrm{t}, J=7.3 \mathrm{~Hz}, 3 \mathrm{H}) ;{ }^{13} \mathrm{C}$ NMR $(101 \mathrm{MHz}$, Chloroform- $d$ ) $\delta$ : 141.0, 140.7, 139.4, 129.4, 127.8, 126.7, $124.9,124.0,123.7,123.2,122.5,120.8,119.6,112.8$, 111.8, 109.7, 109.0, 38.0, 14.1; ESI-HRMS calcd for $\mathrm{C}_{26} \mathrm{H}_{18} \mathrm{Br}_{2} \mathrm{~N}_{2} \quad$ 517.9816, found 517.9815.

3',6'-二溴-9-苯基-3,9'-二咔唑(5s): 棕色固体, 产率 35\%. m.p. 203 204 ${ }^{\circ} \mathrm{C}$; ${ }^{1} \mathrm{H}$ NMR (400 MHz, Chloroform-d) $\delta: 8.23(\mathrm{~d}, J=1.9 \mathrm{~Hz}, 2 \mathrm{H}), 8.21(\mathrm{~d}, J=1.9 \mathrm{~Hz}$, $1 \mathrm{H}), 8.10(\mathrm{~d}, J=7.9 \mathrm{~Hz}, 1 \mathrm{H}), 7.72 \sim 7.62(\mathrm{~m}, 4 \mathrm{H}), 7.61 \sim$ 7.47 (m, 6H), 7.43 (dd, $J=8.6,2.0 \mathrm{~Hz}, 1 \mathrm{H}), 7.33$ (ddd, $J=$ 8.1, 5.0, $3.0 \mathrm{~Hz}, 1 \mathrm{H}), 7.24$ (d, $J=8.8 \mathrm{~Hz}, 2 \mathrm{H}) ;{ }^{13} \mathrm{C} \mathrm{NMR}$ (101 MHz, Chloroform- $d$ ) $\delta: 141.7,140.9,140.3,137.3$, $130.2,129.4,128.9,128.1,127.3,127.0,125.2,124.5$, $123.7,123.3,122.8,120.7,120.6,119.4,112.9,111.7$, 111.2, 110.4; ESI-HRMS calcd for $\mathrm{C}_{30} \mathrm{H}_{18} \mathrm{Br}_{2} \mathrm{~N}_{2} 565.9816$, found 565.9819 .

3',6,6'-三澳-9-苯基-3,9'-二咔唑(5t): 棕色固体, 产 率 42\%. m.p. $255 \sim 256{ }^{\circ} \mathrm{C} ;{ }^{1} \mathrm{H}$ NMR $(400 \mathrm{MHz}$, Chloroform- $d) \delta: 8.22(\mathrm{~s}, 3 \mathrm{H}), 8.16(\mathrm{~s}, 1 \mathrm{H}), 7.72 \sim 7.42(\mathrm{~m}, 10 \mathrm{H})$, $7.33(\mathrm{~d}, J=8.8 \mathrm{~Hz}, 1 \mathrm{H}), 7.22(\mathrm{~d}, J=8.7 \mathrm{~Hz}, 2 \mathrm{H}) ;{ }^{13} \mathrm{C}$ NMR (101 MHz, Chloroform- $d$ ) $\delta$ : 140.8, 140.7, 140.5, $136.9,130.4,129.7,129.5,129.4,128.5,127.2,125.9$, $124.5,123.9,123.5,123.4,123.3,119.5,113.4,113.0$, 111.9, 111.6, 111.5; ESI-HRMS calcd for $\mathrm{C}_{30} \mathrm{H}_{17} \mathrm{Br}_{3} \mathrm{~N}_{2}$ 643.8921, found 643.8922 . 
辅助材料(Supporting Information) 化合物 3a $\sim 3 \mathbf{f}$ 和 5aa $\sim 5 \mathbf{t}$ 的 ${ }^{1} \mathrm{H}$ NMR 和 ${ }^{13} \mathrm{C}$ NMR 谱图. 这些材料可以免 费从本刊网站(http://sioc-journal.cn/)上下载.

\section{References}

[1] (a) Knölker, H.-J.; Reddy, K. R. Chem. Rev. 2002, 102, 4303. (b) Schmidt, A. W.; Reddy, K. R.; Knölker, H.-J. Chem. Rev. 2012, 112,3193 .

[2] (a) Morin, J.-F.; Leclerc, M.; Adès D.; Siove, A. Macromol. Rapid Commun. 2005, 26, 761 .

(b) Zhang, F.-F.; Zhou, C.-H.; Yan, J.-P. Chin. J. Org. Chem. 2010, 30, 783 (in Chinese).

(张飞飞, 周成合, 颜建平, 有机化学, 2010, 30, 783.)

(c) Su, Y.-M.; Lin, H.-J.; Li, W.-M. Prog. Chem. 2015, 27, 1384 (in Chinese).

(苏玉苗，林海娟，李文木，化学进展, 2015, 27, 1384.)

(d) Sadiq, Z.; Hussain, E. A.; Naz, S. Mini-Rev. Org. Chem. 2017, 14, 469.

[3] (a) Ley, S. V.; Thomas A. W. Angew. Chem., Int. Ed. 2003, 42, 5400 .

(b) Beletskaya, I. P.; Cheprakov, A. V. Coord. Chem. Rev. 2004, $248,2337$.

(c) Hartwig, J. F. Acc. Chem. Res. 2008, 41, 1534.

(d) Bariwalab, J.; Eycken E. V. Chem. Soc. Rev. 2013, 42, 9283.

(e) Heravi, M. M.; Kheilkordi, Z.; Zadsirjan, V.; Heydari M.; Malmir, J. Organomet. Chem. 2018, 861, 17.

[4] (a) Zhdankin, V. V.; Stang, P. J. Chem. Rev. 2002, 102, 2523.

(b) Zhdankin, V. V.; Stang, P. J. Chem. Rev. 2008, 108, 5299.

(c) Yoshimura, A.; Zhdankin, V. V. Chem. Rev. 2016, 116, 3328.

(d) Duan, Y.-N.; Jiang, S.; Han, Y.-C.; Sun, B.; Zhang, C. Chin. J. Org. Chem. 2016, 36, 1973 (in Chinese).

(段亚南, 姜山, 韩永超, 孙博, 张弛, 有机化学, 2016, 36, 1973.)
[5] (a) Zhdankin, V. V. Curr. Org. Synth. 2005, 2, 121.

(b) Brand, J. P.; González, D. F.; Nicolai, S.; Waser, J. Chem. Commun. 2011, 47, 102.

(c) Waser, J. Synlett 2016, 27, 2761.

(d) Li, Y.-F.; Hari, D. P.; Vita, M. V.; Waser, J. Angew. Chem., Int. Ed. 2016, 55, 4436.

(e) Hari, D. P.; Caramenti P.; Waser, J. Acc. Chem. Res. 2018, 51, 3212 .

[6] (a) Kieltsch, I.; Eisenberger, P.; Togni, A. Angew. Chem., Int. Ed. 2007, 46, 754 .

(b) Parsons, A. T.; Buchwald, S. L. Angew. Chem., Int. Ed. 2011, 50, 9120 .

(c) Mejía, E.; Togni, A. ACS Catal. 2012, 2, 521.

[7] (a) Brand, P. J.; Charpentier, J.; Waser J. Angew. Chem., Int. Ed. 2010, 48, 9346.

(b) Liu, X.; Wang, Z.; Cheng, X. J. Am. Chem. Soc. 2012, 134, 14330 .

(c) Finkbeiner, P.; Kloeckner, U.; Nachtsheim, B. J. Angew. Chem., Int. Ed. 2015, 54, 4949.

[8] (a) Deng, Q.-H.; Bleith, T.; Wadepohl, H.; Dade, L. H. J. Am Chem. Soc. 2013, 135, 5356.

(b) Sharma, A.; Hartwig, J. F. Nature 2015, 46, 600 .

[9] Kiyokawa, K.; Kosaka, T.; Kojima, T.; Minakata, S. Angew. Chem., Int. Ed. 2015, 54, 13719.

[10] (a) Wang, H.; Zhang, D.; Sheng, H.; Bolm, C. J. Org. Chem. 2017, $82,11854$.

(a) Wang, H.; Zhang, D.; Sheng, H.; Bolm, C. Chem.-Eur. J. 2018, 24, 14942.

[11] Lan, T.-L.; Qin, H.-J.; Chen, W.-T.; Liu, W.; Chen, C. Chin. Chem. Lett. 2019, DOI:10.1016/j.cclet.2019.07.031.

[12] (a) Cai, S.-J.; Chen, C.; Sun, Z.-L.; Xi, C.-J. Chem. Commun. 2013, $49,4552$.

(b) Gao, X.-Y.; Geng, Y.; Han, S.-J.; Liang, A.-P.; Li, J.-Y.; Zou, D.-P.; Wu, Y.-S.; Wu, Y.-J. Org. Lett. 2018, 20, 3732.

[13] Hsiao, S.-H.; Wang, H.-M. RSC Adv. 2016, 6, 43470 